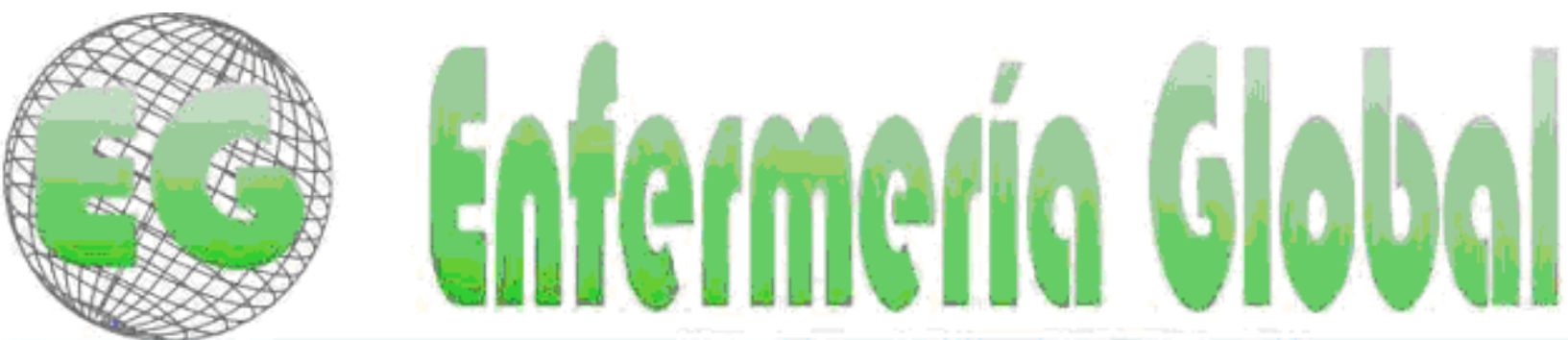

$\mathrm{N}^{\circ} 18$

Riovista electrônica cuatrimestral de Enfermeria

Febrero 2010

www.um.es/egloball

CLÍNICA

\title{
INTERVENCIÓN DE ENFERMERÍA DURANTE LA CLÍNICA PRENATAL Y CONOCIMIENTO SOBRE RIESGOS FISIOLÓGICOS DE LA ADOLESCENTE EMBARAZADA
}

NURSING INTERVENTION DURING PRENATAL CLINIC AND KNOWLEDGE ABOUT THE PHYSIOLOGICAL RISKS OF PREGNANT TEENAGERS

\begin{abstract}
${ }^{*}$ Colmenares, Z., " ${ }^{* *}$ Montero, L., " Reina, R., " ${ }^{* *}$ González, Z.
*Docente Universidad de Carabobo, Máster en Ciencias de la Enfermería, Mención Salud reproductiva. Especialista en Cuidados intensivos del Niño y Neonato, ${ }^{* *}$ Docente Universidad de Carabobo, Magíster en Enfermería Materno Infantil Mención Obstetricia. ${ }^{* * *}$ MgSc en Enfermería Materno Infantil Mención Obstetricia, Área Clínica de Cuidados del niño con problemas de salud. Guarico. Venezuela.
\end{abstract}

Palabras clave: Enfermería Clínica prenatal, conocimiento, adolescente embarazada

Keywords: Prenatal Nursing Clinic, Knowledge, and Pregnant Teenager.

\section{RESUMEN}

El presente estudio tuvo como objetivo determinar la relación que existe entre la intervención de la enfermera en la clínica prenatal y el conocimiento de la embarazada sobre los riesgos fisiológicos. Metodológicamente correspondió a un estudio de campo descriptivo correlacional, se realizó en los ambulatorios urbanos de San Juan de los Morros, Estado Guárico. La muestra estuvo conformada por dos grupos, con muestreo no probabilística por conveniencia, conformado por las 16 enfermeras de los ambulatorios y 41 adolescentes embarazadas. La recolección de información fue a través de dos instrumentos, uno aplicado a las enfermeras con 42 ítems y el otro para las adolescentes con 29 ítems, para ambos instrumentos se les aplicó como índice de confiabilidad el Alfa de Crombach, el cual arrojó un coeficiente de 0,93 y 0,79 respectivamente, lo que demostró un alto índice de confiabilidad para ser aplicado a la muestra final antes descrita.

Entre las conclusiones relevantes está que no existe relación estadísticamente significativa entre la intervención de la enfermera en la clínica prenatal y el conocimiento de la adolescente embarazada sobre los riesgos fisiológicos; además, se plantea que no existe relación estadísticamente significativa entre la intervención de la enfermera en la clínica prenatal en su función asistencial y el conocimiento de la adolescente embarazada sobre los riesgos fisiológicos; pero existe relación estadísticamente significativa entre la intervención de la enfermera en la clínica prenatal en sus función docente y el conocimiento de la adolescente embarazada sobre los riesgos fisiológicos. Por lo tanto, se recomienda desarrollar actividades educativas, en base a factores de riesgo fisiológico, así mismo evaluar la habilidad de la madre para aplicar el aprendizaje obtenido durante las sesiones educativas 


\section{ABSTRACT}

This study aims to: Identify the relationship between the intervention of the nurse in the prenatal clinic and awareness of pregnant women as to the physiological risks. Methodologically it corresponds to a descriptive correlated field study; it was conducted in urban clinics in San Juan de Los Morros, Guarico State (Venezuela). The sample consisted of two groups, with non-probabilistic convenience sample comprised of 16 nurses from clinics and 41 pregnant teenagers. The information was collected through two instruments, one applied to nurses with 42 items and the other for teenagers with 29 items. For both instruments the Alpha Crombach was applied as an index of reliability, which gives a coefficient of 0.93 and 0.79 respectively, which showed a high level of reliability to be applied to the sample above.

Among the relevant findings is that there is no statistically significant relationship between the intervention of the nurse in the prenatal clinic and the pregnant teenager's awareness of the physiological risks. Also, it is argued that there is no statistically significant relationship between the intervention of the nurse in the prenatal clinic in their role of caregiver and the pregnant teenager's awareness of the physiological risks. However, there is a statistically significant relationship between the intervention of the nurse in the prenatal clinic in their role as teacher, and the pregnant teenager's knowledge of the physiological risks. Therefore, it is recommended that educational activities are developed, based on physiological risk factors, and also assess the ability of mother to apply the learning gained during the educational sessions.

\section{INTRODUCCIÓN}

La contribución de la enfermería hacia la mujer embarazada es educar y fortalecer en ella la conciencia del valor de la salud; proveer el cuidado, aplicar procedimientos de intervención y evaluar los resultados. Por lo tanto, la consulta prenatal consiste en un conjunto de actividades y procedimientos que permiten la vigilancia adecuada de la evolución del embarazo, que se concreta en entrevistas o visitas programadas con el equipo de salud, a fin de controlar la evolución del embarazo y obtener una adecuada preparación para el parto y la crianza del recién nacido con la finalidad de disminuir los riesgos de este proceso fisiológico.

Cabe destacar que el objetivo de la salud perinatal es la salud de la madre y el hijo, poniendo énfasis en fomentar una maternidad sin riesgos. De esta manera, se incluyen el control y la vigilancia del embarazo, a fin de identificar oportunamente los riesgos que puedan alterar el curso fisiológico del mismo, así como el de referir los casos de embarazos de alto riesgo a una atención médica específica, especializada y eficaz. (1)

La participación social es una acción fundamental que compete a la enfermera(o), y a la vez es una responsabilidad de las comunidades, que participan a través de grupos, agentes y personas de la comunidad, llamados también comités de salud, que deben involucrarse en acciones que contribuyan en la mejora de aquellos aspectos de la vida cotidiana, la cultura y la actividad política relacionados con la salud.

De allí, que la enfermería implica un encuentro especial de personas humanas, que ocurre como respuesta a una necesidad de cuidado que emerge durante el proceso de gestación. Es una forma particular de relación intersubjetiva, en la cual los participantes (equipo de profesionales en obstetricia, enfermería y enfermería obstétrica, mujer embarazada y familia) son interdependientes, pues participan necesariamente en cada uno de los acontecimientos, sin embargo son independientes y singulares. (2) 
Al surgir el concepto de adolescencia, las perspectivas y condiciones de la mujer y del hombre cambian de manera casi radical tanto desde los campos sociales como individuales. El embarazo en la adolescencia es considerado un problema desde diferentes ópticas, ya que implica riesgo de mortalidad materna, menores oportunidades de educación y, en ocasiones, el abandono total de los estudios, así como el fortalecimiento del círculo de la pobreza y un mayor riesgo de daño y mortalidad infantil.

En el mundo ocurren anualmente 15 millones de partos en adolescentes, de los cuales el $80 \%$ son pertenecientes a países en vías de desarrollo. Diversos estudios indican que más del 50 por ciento de las y los jóvenes menores de 17 años son sexualmente activos (1). Este fenómeno es denominado sexualidad temprana en numerosos documentos, e implica riesgos de un embarazo, deseado o indeseado, con consecuencias negativas a corto y largo plazo para las mujeres adolescentes.

El embarazo en la adolescencia se constituye, por lo tanto, en una grave amenaza de riesgo para el desarrollo psicosocial de la mujer embarazada, pues a lo largo de la historia se observa que el embarazo en la adolescencia siempre existió. Sin olvidarse, por otro lado, que abuelas y bisabuelas se casaban a los 13 años y eran madres a los 14 años, en gran parte de la América Latina(4). Las adolescentes son vistas de forma diferente por la sociedad y por su propia familia, porque su cuerpo comienza a prepararse para la procreación, para un posible embarazo, sin querer significar con esto que tenga condiciones para asumirlo socialmente. Por otro lado, es importante recordar que la humanización en el feto surge como parte de su relación cultural y comienza en el embarazo cuando comienza a ser deseado por su madre a partir de sus sentimientos y reflexiones, en tal sentido la enfermera durante el embarazo en la adolescencia debe fortalecer las sesiones educativas para afianzar el autocuidado de la adolescente

En la adolescencia, de forma muy particular, se descubre, claramente, la expresión del amor erótico. Esta tendencia a ser amoroso, característica de la adolescencia, es vivida durante su proceso de crecimiento y desarrollo, en una relación amorosa con la protección de los padres y de la propia sociedad. Por eso, los niños deben ser protegidos, pues se constituyen en un bien precioso para la propia sociedad. Ellos deben ser protegidos de la falta de amor o del falso uso del amor, ofreciéndoles desde su fase de vida intrauterina la oportunidad de vivenciar un proceso amoroso. (8)

Al entrar en la adolescencia, especialmente la mujer a partir de la menstruación, como referencia sexual, señala biológicamente que está preparada para otra expresión del amor, el amor erótico, y que puede correr el riesgo de quedar embarazada. Como opina (9), es una concreción de sueños y de fantasías, y el olvido de la falta de afecto es la prueba de la heterosexualidad: "Yo soy formal, sentí placer, no soy impotente o frígida sexual". Es ir al encuentro del amor en su plenitud, es amar y ser amada.

La adolescente embarazada para tener orientación de los riesgos durante el embarazo analiza las facultades cognitivas y su capacidad de captar la realidad en sus diversas manifestaciones es más que una disciplina filosófica general, esta no se ocupa de una verdad particular de algún campo de la ciencia, sino que se ocupa del cuerpo de las verdades cognoscibles. (10)

Por tanto, el conocimiento en la adolescencia debe partir del principio que por su explicación sistemática se determina el modo de conocer los aspectos de la realidad en su salud reproductiva, el cual abarca desde su reflejo superficial hasta el dominio de las leyes que rigen sus fenómenos a través del conocimiento.

Los adolescentes disponen de una valiosa contribución para dar a la sociedad y están 
abiertos a las modificaciones necesarias para el desarrollo de la salud. Además, están dispuestos a adoptar nuevas actitudes en lo que se refiere a la función social y personal, especialmente la mujer, en el establecimiento de la igualdad en sus relaciones (11). La adolescencia es un período de intensos cambios físicos y psico-sociales, que usualmente comienzan y pueden terminar en la segunda década de la vida" (13.). Los cambios físicos más notables en la adolescencia son de orden endocrino y la evolución de los caracteres sexuales en la pubertad en ambos sexos. La cantidad y duración tienen variaciones individuales. Estos cambios son importantes tomarlos en cuenta para prever atención de salud integral y apreciar las diferencias entre las variaciones normales y las anormalidades en el proceso de crecimiento y desarrollo (5).

El embarazo en la adolescente es "el que ocurre dentro de los dos años de edad ginecológica, entendiéndose por tal el tiempo transcurrido desde la menarquía, y/o cuando la adolescente es aún dependiente de su núcleo familiar de origen". Tal embarazo, además de considerarlo un "problema", exige aplicar terapéuticas que aporten soluciones sin permitir implementar acciones preventivas adecuadas (11).Por ello es conveniente fundamentarlo dentro del marco de la "salud integral del adolescente". Esto permite abarcar todos los embarazos que ocurran a esta edad; adecuar las acciones preventivas dentro de la promoción de la salud; brindar asistencia integral a cada madre adolescente, a sus hijos y parejas y aportar elementos para el desarrollo de las potencialidades de los adolescentes.

Por todo ello, el embarazo en adolescentes necesita un abordaje integral biopsicosocial por un equipo interdisciplinario capacitado en la atención de adolescentes y en este aspecto específico de la maternidad - paternidad. De acuerdo a este planteamiento es importante abordar la orientación para que la adolescente identifique precozmente los riesgos que pudieran estar presentes en la salud de la adolescente embarazada, para efecto del estudio. De acuerdo a la Organización Mundial de la Salud y la Federación Internacional de Planificación de la Familia (1), menciona que en el embarazo precoz los factores de riesgo se clasifican en: 1. Personales: edad de la menarquia, deseo de independencia, ignorancia de su anatomía y fisiología, desconocimientos de métodos preventivos, soledad y baja autoestima, embarazo anterior, uso y abuso de drogas. 2. Familiares: Inestabilidad familiar, padres con enfermedad crónica, ejemplo familiar de embarazo precoz, falta de comunicación, liberación de costumbres, estrato social, presión grupal o pareja, carencia de oportunidades reales, influencia de los medios de comunicación social, falta de políticas coherentes, abuso sexual, prostitución y pornografía en adolescentes.

En base a lo anterior, se deduce que la mayoría de las madres adolescentes pertenecen a sectores sociales muy desprotegidos. En las circunstancias en que estas jóvenes crecen, el proceso de adolescencia tiene características particulares. Un sentimiento presente en las adolescentes durante su embarazo es el de verse privadas de las actividades que corresponden a su edad y confundiendo su rol dentro del grupo, pasando a ser una "hijamadre". En tal sentido los riesgos fisiológicos de la gestante incluyen primordialmente la Conservación de la salud en el embarazo: el embarazo debe ser una experiencia normal, sana y feliz para la mujer, si su salud general es buena, no hay razón por la cual la gestación produzca síntomas físicos o emocionales que interfieran en su capacidad de actuar y participar en actividades corrientes, se estimula a la gestante a que no interrumpa sus ocupaciones y hábitos. (12)

La adolescente tiene poca conciencia de salud, resultándole muy difícil asumir su propio cuidado debido a las circunstancias en que ocurrió el embarazo y las dificultades que éste le plantea. No tiene tiempo para pensar que debe concurrir regularmente a la consulta, ni comprende la importancia de los estudios complementarios, interpretándolos como castigo. 
Por ello hay que explicarle muy cuidadosamente para qué sirven y cómo se los realizarán, de allí que modificar el estilo de vida de la adolescente embarazada sea una decisión personal y una forma particular de vivir de la persona, la cual puede estar determinada por las circunstancias ambientales. La elección del estilo de vida determinará en el individuo el comportamiento a seguir con respecto a sus hábitos alimentarios, de recreación, tabáquicos, alcohólicos, y de relaciones interpersonales. De tal manera que cada individuo asume según su decisión su comportamiento en cuidado de su salud.

\section{MATERIALES Y MÉTODOS}

Se utilizó para el estudio una metodología de investigación cuantitativa, con un diseño No experimental, Transversal, descriptivo; La Población objeto de estudio estuvo conformada por las enfermeras y las adolescentes embarazadas de los Nueve Ambulatorios Urbanos del Distrito Sanitario № 1 del Estado Guárico, Venezuela. Una población de 22 Enfermeras(os) del área comunitaria, y una muestra probabilística al azar simple de 16 enfermeras(os) que representó el $72,72 \%$ de la población, en la adolescente embarazada de una población de 168 adolescentes embarazadas se tomó una muestra al azar simple de 41 adolescentes que representó el $24,40 \%$. Se recolectó la información a través de dos instrumentos, tipo cuestionario con escala Lickert donde el instrumento dirigido a la enfermera contó con 42 ítems con una confiabilidad de 0,93 y para la adolescente embarazada 29 ítems con una confiabilidad de 0,79 obtenido para ambos instrumentos mediante el uso del alfa de Crombach. La validación del instrumento se realizó mediante la aplicación de los siguientes procedimientos: validación por juicio de expertos en contenido, tomando en cuenta el tipo de estudio, objetivos y operacionalización de la variable. Se realizó prueba piloto a 10 enfermeras con un índice de confiabilidad de 0,93 mediante el alfa de Crombach y 10 adolescentes embarazadas con un índice de confiabilidad de 0,79 según el alfa de Crombach; estas muestras fueron diferentes a las de la prueba final con las características de la población a objeto de estudio, para determinar los datos se analizaron y transfirieron a una matriz de cuadros de frecuencias, aplicándole a cada cuadro de asociación la media, desviación Standard y la prueba T. Una vez realizados estos cálculos, se procedió a establecer si los porcentajes obtenidos para los factores y las variables seguían una distribución normal, mediante la prueba de Kolmogorv Smirnov, evaluada ésta mediante la prueba de la u de Mann Whitney.

\section{ANÁLISIS DE RESULTADOS:}



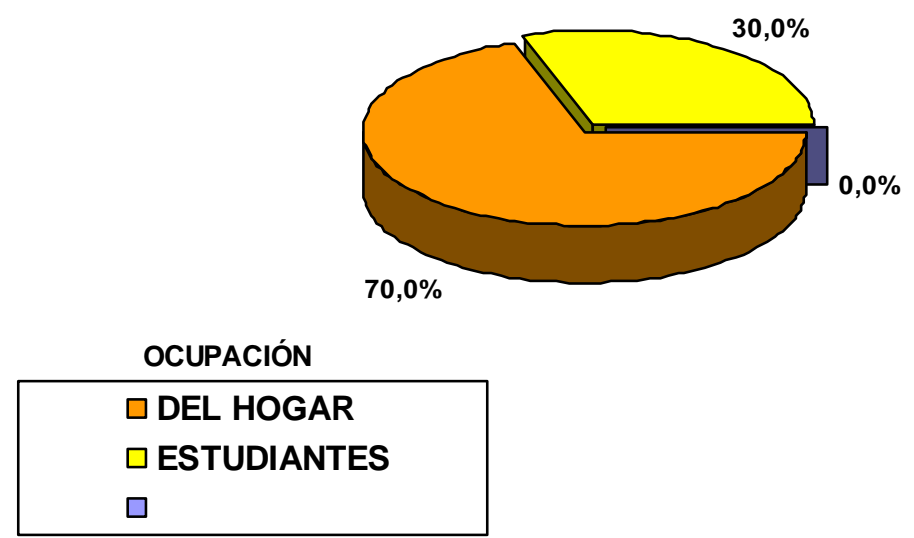

Gráfico 1 Distribución de las Adolescentes según su ocupación. Ambulatorios Urbanos del Parroquia Juan German Roscio. San Juan de los Morros. Octubre. (2008

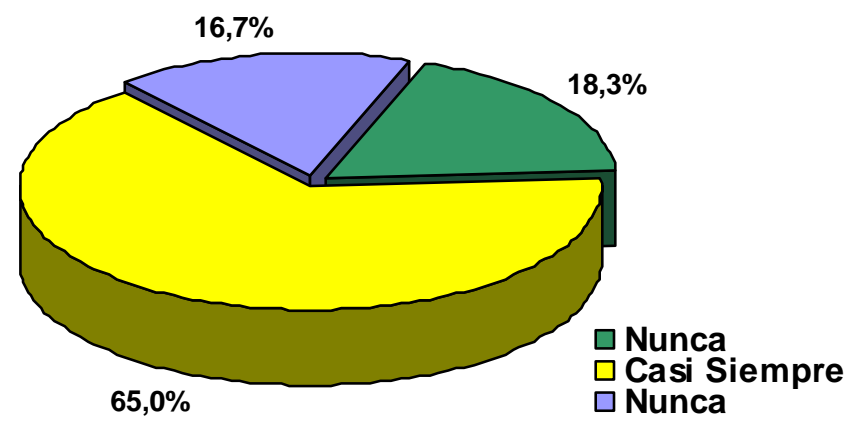

Gráfico 2. Distribución de las adolescentes embarazadas investigadas según ocurrencia consolidada de la variable conocimiento sobre los riesgos fisiológicos. Parroquia Juan German Roscio. San Juan de los Morros. Mayo. 2008.

El 58,5\% de los integrantes del grupo de adolescentes embarazadas investigadas, ubicaron sus respuestas con respecto a la variable conocimiento sobre los riesgos fisiológicos, en la ocurrencia consolidada de casi siempre, correspondiendo el restante $41,5 \%$ de las mismas a la ocurrencia consolidada de siempre. 


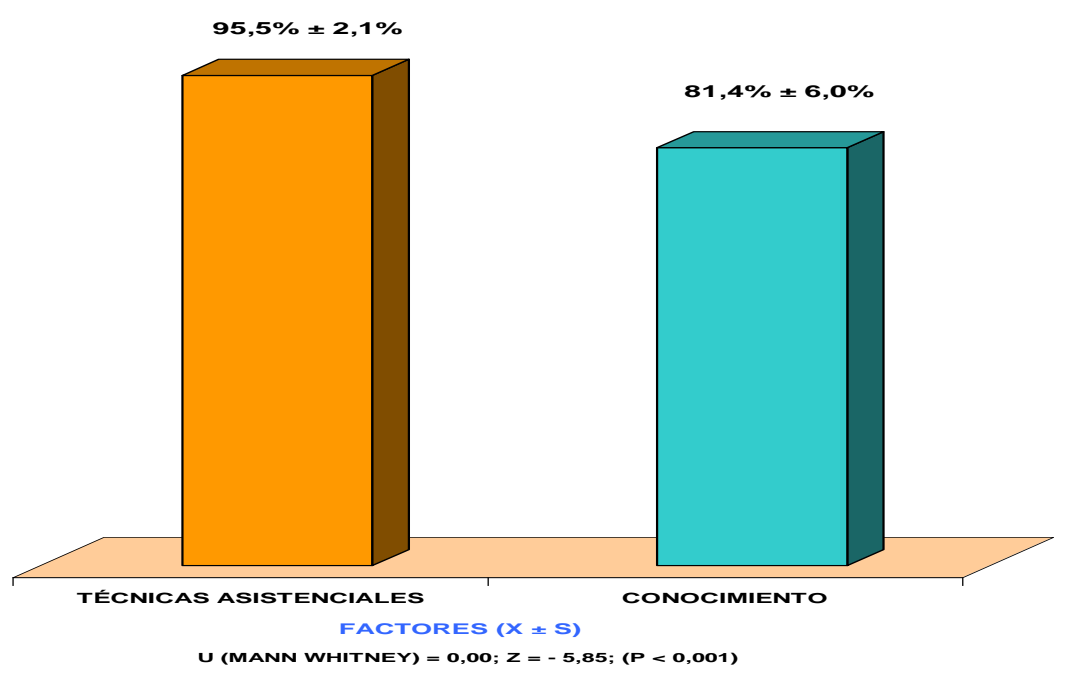

Gráfico 3. Valores promedios (x) con desviación estándar (s) expresados porcentualmente de la ocurrencia del factor técnicas asistenciales y de opinión del factor conocimiento según respuestas del personal de enfermería y de las adolescentes embarazadas respectivamente. Ambulatorios Urbanos del Parroquia Juan German Roscio. San Juan de los Morros. Octubre. 2008

El valor medio de la ocurrencia expresada por el personal de enfermería como porcentaje del nivel ideal $(100,0 \%)$ del factor técnicas asistenciales fue 95,5\% con desviación estándar de $2,1 \%$, muy superior a la opinión expresada en porcentaje promedio del factor conocimiento del ideal esperado $(100,0 \%)$ de las adolescentes investigadas; cuya cifra media alcanzó un valor de $81,4 \%$ con desviación estándar de 6,0\%. Puesto que no era posible comparar los promedios, por ser la distribución de los porcentajes de las adolescentes diferente a la normal, se aplicó la prueba $U$ (de Mann Whitney) para establecer si existe o no diferencias significativas, en los rangos que ocupan los valores porcentuales de ambos factores.

Esto pudiera deberse a que la enfermera entre sus funciones técnicas esencial tomado en cuenta para el estudio es la visita domiciliaria como seguimiento de casos, a nivel comunitario y pudiera no estar llevándose, lo que no permite brindar una interacción humana de carácter transpersonal, para apoyar a la adolescente embarazada durante el embarazo, fortaleciendo las relaciones del grupo familiar y de la adolescente. Esta situación predispone a la adolescente a asumir conductas inapropiadas en la evolución de su embarazo puesto que el conocimiento acerca de los riesgos del embarazo pudiera no estar claro para esta adolescente, la función asistencial de Enfermería para la adolescente en estado de embarazo es escalonado, consciente, y envuelve evolución y reflexión, pues, además de ayudar a las personas para que estas tengan un mayor conocimiento de sí mismas con una óptica universal. En un sentido más profundo, "concientización" no es más que mirarse a uno mismo (11). Es, también, un compromiso social que envuelve el desarrollo de Enfermería y de la adolescente embarazada en sus múltiples dimensiones, transformando el proceso energético en un compromiso social con la vida (1). 


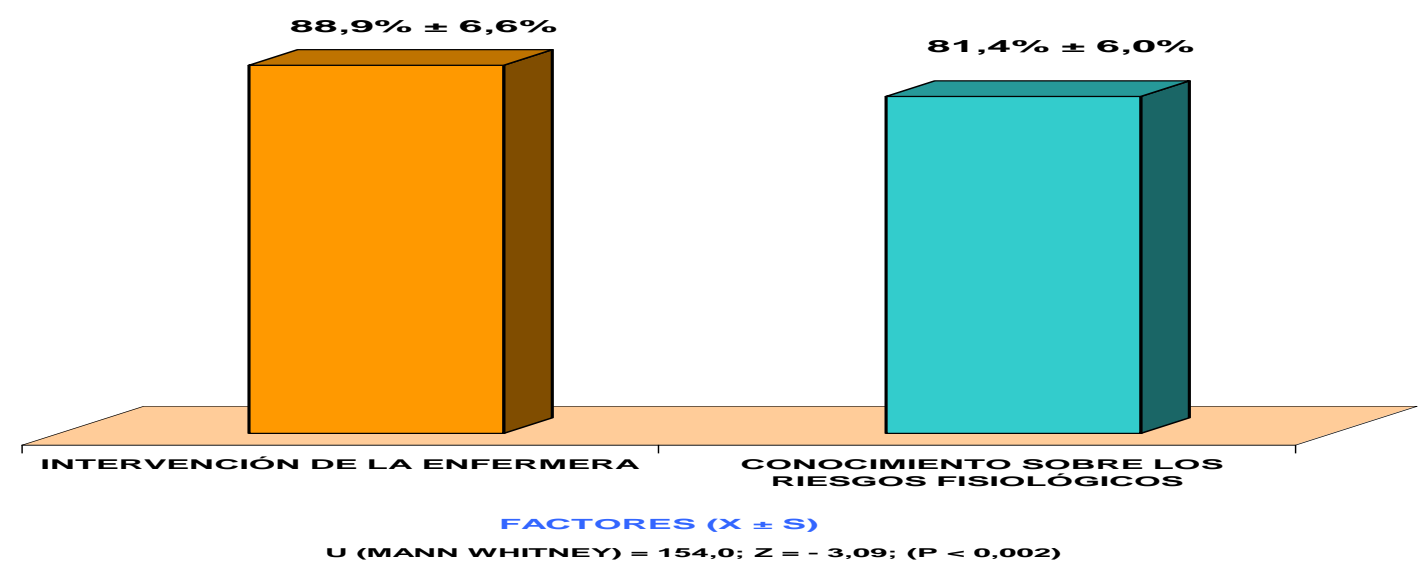

Gráfico 4. Valores promedios $(\mathrm{x})$ con desviación estándar (s) expresados porcentualmente de la ocurrencia del factor intervención docente y de opinión del factor conocimiento según respuestas del personal de enfermería y de las adolescentes embarazadas respectivamente. Ambulatorios Urbanos de la Parroquia Juan German Roscio. San Juan de los Morros. Octubre. 2008

En el gráfico 4, se evidencia, el valor medio de la ocurrencia expresada por el personal de enfermería como porcentaje del nivel ideal $(100,0 \%)$ de la variable intervención de la enfermera fue 88,9\% con desviación estándar de 6,6\%, muy superior a la opinión expresada en porcentaje promedio del factor conocimiento sobre los riesgo fisiológicos del ideal esperado $(100,0 \%)$ de las adolescentes investigadas; cuya cifra media alcanzó un valor de $81,4 \%$ con desviación estándar de $6,0 \%$. Puesto que no era posible comparar los promedios, por ser la distribución de los porcentajes de las adolescentes diferente a la normal, se aplicó la prueba u (de Mann Whitney) para establecer si existe o no diferencias significativas, en los rangos que ocupan los valores porcentuales de ambos factores. Los resultados de la prueba indican que existe una discrepancia significativa $(p<0,002)$, entre los órdenes que ocupan los valores porcentuales en ambas distribuciones (expresados en términos de suma de rangos y rangos medios para la comparación respectiva de la prueba), por lo que se puede asegurar que no hay correspondencia o asociación significativa entre los niveles porcentuales de ocurrencia de la variable intervención de la enfermera y los niveles porcentuales de opinión del factor conocimiento sobre los riesgos fisiológicos de las adolescentes investigadas. Es decir, se debe rechazar la hipótesis de que existe relación estadísticamente significativa entre la intervención de la enfermera en la clínica prenatal y el conocimiento de la adolescente embarazada sobre los riesgos fisiológicos. Esto es debido a que el efecto que tiene el factor técnicas asistenciales es superior al de la intervención docente, aunque este último como factor se haya relacionado o asociado significativamente al conocimiento de las adolescentes embarazadas.

\section{CONCLUSIONES}

1.- Se rechaza la hipótesis general de trabajo y se acepta la hipótesis general nula que plantea que no existe relación estadísticamente significativa entre la intervención de la Enfermera en la clínica prenatal y el conocimiento de la adolescente embarazada sobre los riesgos fisiológicos. 
2.- Cabe destacar, que se rechaza la hipótesis especifica oㅜ 1 y se acepta la hipótesis específica nula ํo 2 que plantea que no existe relación estadísticamente significativa entre la intervención de la enfermera en la clínica prenatal en su función asistencial y el conocimiento de la adolescente embarazada sobre los riesgos fisiológicos

3.- Por consiguiente, se acepta la hipótesis especifica oㅡ 2 que plantea que existe relación estadísticamente significativa entre la intervención de la enfermera en la clínica prenatal en sus función docente y el conocimiento de la adolescente embarazada sobre los riesgos fisiológicos.

\section{RECOMENDACIONES:}

1.- Se deben desarrollar actividades educativas, según las necesidades identificadas en base a factores de riesgo fisiológico, así mismo evaluará la habilidad de la madre para aplicar el aprendizaje obtenido durante las sesiones educativas

2.- Desarrollar técnicas de trabajo en familia para apoyar los sentimientos de las adolescentes embarazadas y/o promover conductas y actitudes positivas hacia el neonato y los (as) adolescentes.

3.- Fortalecer la educación para la salud en las adolescentes en control, así como su grupo familiar, capacitándolos (as) sobre el conocimiento de los factores de riesgos durante el embarazo, conserjería en lactancia materna, cuidado durante el embarazo, parto, puerperio y cuidados del recién nacido, así como también promover y/o fortalecer el proceso de vinculación afectiva madre-hijo y/o darle una preparación psicofísica (psicoprofiláctico), para que tenga un parto tranquilo con feliz término.

4.- Promover la utilización de metodologías participativas durante las sesiones educativas de las adolescentes embarazadas para favorecer creencias de conductas negativas hacia su autocuidado a través de juegos educativos, sociodramas, lluvia de ideas, discusiones grupales, collage y cualquier otra metodología según la creatividad del personal, donde ellos (as) puedan intercambiar conocimientos y experiencias relacionadas con el embarazo, parto, puerperio y cuidados del recién nacido.

5.- Estimular, promover y facilitar la participación de la pareja durante todo el proceso, brindándole apoyo psicológico a ambos.

6.- Presentar los resultados en eventos científicos, locales e internacionales, como aporte al conocimiento especifico de la disciplina y fortalecer las líneas de investigación en enfermería en el Estado Guarico.

7.- Entregar una copia del presente estudio a las Gerencias de los Ambulatorios, para que se cree la consulta de atención integral a la adolescente embarazada en los nueve ambulatorios estudiados con la finalidad de ir mostrando este modelo al resto del Estado Guarico.

\section{REFERENCIAS BIBLIOGRÁFICAS}

1.- Organización Mundial de la Salud. Materna en América Latina. Boletín Epidemiológico. 2.008. Washington.

2.- Desser. Embarazo en adolescente. O.P.S/.O.M.S. Publicación Cientifica. 2.006.

3.- Maturana. El sentido de lo humano. Convivencia, aceptación y creatividad. Santiago. 
4.- Desser. Embarazo en adolescente. O.P.S.O.M.S. Publicación Cientifica. 2.000

5.- Fishbein y Arzen. La adolescente embarazada. Rev. Obstest. Ginecol. Venezuela. 1.975.

6.- Hamilton, M. Asistencia Materna Infantil de Enfermería. México: Interamericana. 1990.

7.- Hernández, Fernández y Baptista Metodología de la Investigación. Editorial Mc Graw Hill. 1.999.

8.- Issler Embarazo en Adolescencia. Revista de Posgrado de la Cátedra Vzla Medicina № 107 - Agosto/2001 Página: 11-23 .2.001.

9.- Maturana. El sentido de lo humano. Convivencia, aceptación y creatividad. Santiago. Ediciones Pedagógicas Chilena 1.991.

10.- Manual de Salud Reproductiva. Programa de Atención Integral de salud para el Adolescente. Normas y Funciones. 2da. Edición. Caracas. 1.999.

11.- Montes Valverde, M. Un Modelo Fundamentado en el Amor para la Asistencia y el Cuidado de las Adolescentes Embarazadas. Cali, Colombia: XYZ. 1999.

12.- Ministerio de Salud y Desarrollo Social Políticas de salud dirigida a la adolescente embarazada. Caracas. 2.000.

13.- Normas del Programa de Atención Integral de Salud para Adolescentes, Dirección Materno Infantil. Publicación MSAS-OPS-OMS-UNICEF. Marzo 1991. Caracas.

14.- Valera. Modelo Fundamentado en el amor para la asistencia y el cuidado de las adolescentes embarazadas. Creaciones Dávalos Bogotá. Colombia 2.003. 